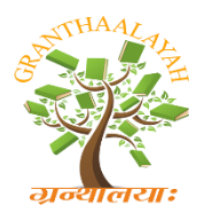

INTERNATIONAL JOURNAL OF RESEARCH GRANTHAALAYAH A knowledge Repository

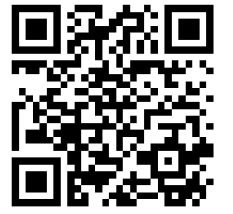

Science

\title{
RESISTANCE PATTERNS OF MULTI-DRUG RESISTANT ESCHERICHIA COLI CAUSING URINARY TRACT INFECTION
}

\author{
Samiya Sabea Khamees ${ }^{* 1}$, Khadija Saeed Ali Ghafir ${ }^{2}$ \\ ${ }^{* 1,2}$ Biology Department, Faculty of Science \& Tobruk University /Libya
}

\begin{abstract}
480 mid-stream urine samples were collected from outpatients and inpatients attending Tobruk Medical Center. 300 samples gave positive culture, 162 of these were Escherichia coli.

The strains sensitivity to 14 antibiotics was tested. High incidence of resistant strains, high degree of association between resistance and complex resistance patterns of the same strains is seen. The strains were resistant to Ampicillin, Penicillin, Erythromycin, Cephalexin and Sulfamethoxazole with $91.9 \%, 90.7 \%, 82.7 \%, 76.5 \%$ and $57.4 \%$ respectively. The strains were sensitive to Neomycin, Oxacillin and Nitrofurtoin with $100 \%$, 99\% and $83 \%$ respectively. A symmetrical result for all strains resistance to indicated pairs of drugs were obtained. Strong association between most antibiotic occurred.
\end{abstract}

Keywords: Escherichia Coli; Antibiotics; Resistance Patterns; Urinary Tract Infection.

Cite This Article: Samiya Sabea Khamees, and Khadija Saeed Ali Ghafir. (2020). "RESISTANCE PATTERNS OF MULTI-DRUG RESISTANT ESCHERICHIA COLI CAUSING URINARY TRACT INFECTION." International Journal of Research - Granthaalayah, 8(4), 153-159. https://doi.org/10.29121/granthaalayah.v8.i4.2020.20.

\section{Introduction}

The large intestine constitutes the main source of E.coli, which is the principle cause of variety infections including urinary tract infection. Urinary Tract Infection (UTI) is one of the most distributed bacterial infection in many developing countries where good sanitation is not maintained Tanzina et al., (2016). It has been reported that up to 15 million person are infected each year worldwide Stam and Norrby, (2001). Antimicrobial resistance has been considered as rising risk both in developed and developing countries (Pfaller; 2000). This risk could be sever in developing countries, where there is no strict information program concerning the use of antibiotics (Jigna and Pratibha, 2012). There are world wide data show that there is increasing resistance among UTI causative agents to the most common prescribed antibiotics, and resistance has emerged even to newest and most efficient antibiotics (Taneja et al., 2008). 
The most important antibiotic resistance risks are reported in Enterobacteraceae for all major antigram negative agents such as beta-lactam, fluoroquinolones and aminoglycosides (Osterblat et al., 2000).

Multidrug resistance in E.coli is of particular concern because it is the most common gramnegative pathogen causing many infections including UTI. In addition, resistant E.coli strains have the ability to exchange antibiotics resistance factor (R-Factor) to other strains of E.coli but also to other gram-negative pathogens (Jiana and Pratibha, 2012).

Because now day's antibiotics resistance has become an important phenomenon due to widespread use of antibiotics by patient without testing antibiogram (Tanziaet al., 2016).

It is obligatory to have knowledge about the organisms causing UTI and there susceptibility to antibiotics. Therefore, the purpose of this study to isolate, and identify E.coli strains from urine samples and determine their susceptibility to common available antibiotics which may help the physician to choose the right treatment for the prevention of UTI.

\section{Materials and Methods}

Mid-stream urine samples were collected from 480 inpatients and outpatients having symptom suggestive of urinary tract infection attending Tobruk Medical Centre - Tobruk - Libya during the one-year period. The specimens were taken on midday and cultured immediately on MacConkey agar and blood agar by standard culture techniques.

Identification of the isolated organism was done on the basis of cultural characteristic and gram staining was performed to confirm the gram-negative bacilli. Apart from colony morphology, the routine biochemical tests were performed using API 20 system for Enterobacteraciae.

The resistance patterns of E.coli isolated strains to 14 antibiotics was determined by using discdiffusing methods and interpreted according to the Nccls (National committee for clinical Laboratory Subcommittee 1990). The following antimicrobial agents were obtained from (Oxoid, UK), Ampicillin, Amoxicillin/calvunic acid, Chloramphenicol, Cefrixon, Cephalexin, Gentamycin, Tetracycline, Nitrofurtoin, Sulfamethoxazole, Erythromycin, Penicillin, Cloxacillin, Oxacillin and Neomycin.

All these tests were performed on Muller-Hinton agar (Oxoid U.k). A 0.05 McFarland Suspension was applied to the plates, which were dried in an incubator at $35 \mathrm{c} \circ$ for 15 minutes, antimicrobial disc was placed on the agar with sterile forceps. The agar plates were incubated at $35 \mathrm{c}^{\circ}$ for $15 \mathrm{hrs}$. The antibiotics effect for each antibiotics was measured as the diameter of the zone of inhibition.

\section{Results and Discussion}

Of the 480 urine samples tested from November 2015 to December 2016, 300 urine samples showed positive growth 162 (54\%) were E.coli.(Table-1). 
Table 1: Species of Gram-negative Bacteria Isolated from Urine Samples

\begin{tabular}{|c|c|c|}
\hline Species of Bacteria & Number & $\%$ \\
\hline Pseudomonas aeruginosa & 26 & 8.666667 \\
\hline Klebsiella pneumonia & 70 & 23.33333 \\
\hline Escherichia coli & 162 & 54 \\
\hline Proteus mirabilis & 42 & 14 \\
\hline Total & 300 & 100 \\
\hline
\end{tabular}

E.coli strains were collectively tested for sensitivity and resistance against 14 antibiotics (Table 2). The antibiotics susceptibility of the isolates showed that E.coli strains were sensitive to Neomycin, Oxacillin and Nitrofurantoin with 100\%, 99\%, and 83\% respectively.

Table 2: Sensitive and Resistance of Escherichia coli Isolated from Urine Samples

\begin{tabular}{|l|c|c|c|c|}
\hline Antibiotic & Resistance(R) & \% & Sensitivity(S) & \% \\
\hline Ampicillin & 149 & 91.97531 & 13 & 8.024691 \\
\hline Amoxicillin/ Clavulanic acid & 54 & 33.33333 & 108 & 66.66667 \\
\hline Chloramphenicol & 64 & 39.50617 & 98 & 60.49383 \\
\hline Cefriaxon & 91 & 56.17284 & 71 & 43.82716 \\
\hline Cephalexin & 124 & 76.54321 & 38 & 23.45679 \\
\hline Gentamycin & 86 & 53.08642 & 76 & 46.91358 \\
\hline Tetracycline & 82 & 50.61728 & 80 & 49.38272 \\
\hline Nitrofurantoin & 27 & 16.66667 & 135 & 83.33333 \\
\hline Sulfamethoxazole & 93 & 57.40741 & 69 & 42.59259 \\
\hline Erythromycin & 134 & 82.71605 & 28 & 17.28395 \\
\hline penicillin & 147 & 90.74074 & 15 & 9.259259 \\
\hline Cloxacillin & 90 & 55.55556 & 72 & 44.44444 \\
\hline Oxacillin & 1 & 0.617284 & 161 & 99.38272 \\
\hline Neomycin & 0 & 0 & 162 & 100 \\
\hline
\end{tabular}

Ampicillin (AMP), Amoxicillin/Clavulanic acid (AMC), Chloramphenicol (C), Cefriaxon (CR), Cephalexin (CL), Gentamycin (CN), Tetracycline (TE), Nitrofurantoin (F), Sulphamethaxazole(SXT), Erythromycin (E), penicillin (P), Cloxacillin (OB), Neomycin (N)

The sensitivity result of E.coli to the aminoglycoside antibiotic Neomycin with100 percentage and Gentamicin $46.9 \%$ was not agreed with the result of Tanzina et al., 2016 as they reported that E.coli strains were sensitive to the aminoglycosides antibiotics gentamycin and Amikacin with $100 \%$. The result of this study showed that E.coli isolates were sensitive to Nitrofurantoin; this was similar to the result of Niranjan and Malini, 2014 and Nalini et al., 2014. However, (Tanzina et al., 2016) reported that E.coli isolates in their study were sensitive to Nitrofurantoin with $93 \%$. Sensitivity of E.coli strains to Nitrofurantoin may indicate the narrow spectrum of activity and limited contact of this antibiotic with bacteria outside the urinary tract (Karlowsky et al., 2002) or probably due to the non-usage of these antibiotics for long period. Nitrofurantoin has been less commonly used in treatment of UTI infection in recent years (Nalini et al., 2014). Other antibiotic, which found effective against E.coli in this study, was Oxacillin, as $99.3 \%$ of E.coli strains were sensitive to this antibiotic. Augmentin (Amoxicillin/calvunic acid) has been known to be efficient 
ISSN- 2350-0530(O), ISSN- 2394-3629(P)

https://doi.org/10.29121/granthaalayah.v8.i4.2020.20

drug against UTI isolates and its resistance was found to be low as we found only $33.3 \%$ of E.coli were resistant. Our result was not in agreement with the results of others (Shafaq et al.,2011; Taneja et al., 2007; Mehrgan and Rahbar 2008) as they reported that their isolates were resistant to Amoxicillin/ calvunic acid with $85 \%, 95 \%$, and $70 \%$ respectively.

The result of this study revealed that E.coli isolates were resistant to Ampicillin and Penicillin with $91.9 \%$ and $90.7 \%$ respectively. In addition, they were resistant to Erythromycin with $82.7 \%$. This result was consistent with other studies carried by Mejbah et al., 2011 and Alessandra et al., 2011.

This high resistance rate may be due to the widespread and prolonged use of these antimicrobial drugs in the world including Libya. High resistance rate also recorded in this study for the first and third generation Cephalosporin antibiotics Cephalexin and ceftriaxone with $76.5 \%$ and 56\% respectively. This result was in agreement with the result of Nalini et al., 2014 concerning Ceftriaxone and different from the result of Tanzina et al., 2016, they reported that E.coli strains were sensitive to Ceftriaxone with 63\%. However, Mohamed, 2010 reported that E.coli isolates were sensitive to cephalexin and ceftriaxone with $20.9 \%$ and $11.7 \%$ respectively.

Tetracycline resistance is increasing in clinical isolates of E.coli. In our study, $50.6 \%$ of E.coli isolates were resistance to this antibiotic and was less than the results of Shfaq et al., 2011 and Noor et al., 2004 as their isolates were resistant to Tetracycline with $95 \%$ and $83.9 \%$ respectively. Although E.coli isolates in this study showed resistance to beta-lactam antibiotics, Ampicillin and Penicillin being the most ineffective drug in addition they showed resistance to Cloxacillin with $55.5 \%$ whereas one of the most effective antibiotics in this study Oxacillin (99.9\%) also belong to the same class. This result is consistent with the result of Rabia et al., 2012 for Imipenem (100\%), Meropenem $99.3 \%$, Piperacillin/Tazobactum 96.8\%.

Table 3: Matrix Indicating Number of Escherichia coli to two Antibiotic at the Same Time.

\begin{tabular}{|l|l|l|l|l|l|l|l|l|l|l|l|l|l|l|}
\hline N & 0 & 0 & 0 & 0 & 0 & 0 & 0 & 0 & 0 & 0 & 0 & 0 & 0 & - \\
\hline OX & 1 & 1 & 0 & 1 & 1 & 0 & 0 & 0 & 1 & 0 & 1 & 1 & - & 0 \\
\hline OB & 77 & 44 & 57 & 66 & 70 & 59 & 39 & 17 & 49 & 85 & 78 & - & 1 & 0 \\
\hline P & 140 & 49 & 57 & 86 & 112 & 74 & 75 & 21 & 84 & 121 & - & 78 & 1 & 0 \\
\hline E & 123 & 50 & 61 & 82 & 101 & 79 & 63 & 22 & 73 & - & 121 & 85 & 0 & 0 \\
\hline SXT & 53 & 23 & 28 & 50 & 77 & 59 & 45 & 18 & - & 73 & 84 & 49 & 1 & 0 \\
\hline F & 25 & 15 & 16 & 15 & 25 & 20 & 16 & - & 18 & 22 & 21 & 17 & 0 & 0 \\
\hline TE & 75 & 29 & 31 & 47 & 65 & 41 & - & 16 & 45 & 63 & 75 & 39 & 0 & 0 \\
\hline CN & 74 & 33 & 42 & 62 & 72 & - & 41 & 20 & 59 & 79 & 74 & 59 & 0 & 0 \\
\hline CL & 117 & 42 & 55 & 91 & - & 72 & 65 & 25 & 77 & 101 & 112 & 70 & 1 & 0 \\
\hline CR & 88 & 43 & 54 & - & 91 & 62 & 47 & 15 & 50 & 82 & 86 & 66 & 1 & 0 \\
\hline C & 57 & 44 & - & 54 & 55 & 42 & 31 & 16 & 28 & 61 & 57 & 57 & 0 & 0 \\
\hline AMC & 49 & - & 44 & 43 & 42 & 33 & 29 & 15 & 23 & 50 & 49 & 44 & 1 & 0 \\
\hline AMP & - & 49 & 57 & 88 & 117 & 74 & 75 & 25 & 53 & 123 & 140 & 77 & 1 & 0 \\
\hline & AMP & AMC & C & CR & CL & CN & TE & F & SXT & E & P & OB & OX & N \\
\hline
\end{tabular}

Ampicillin ( AMP), Amoxicillin/Clavulanic acid ( AMC), Chloramphenicol(C),Cefriaxon (CR), Cephalexin(CL), Gentamycin $(\mathrm{CN})$, Tetracycline(TE), Nitrofurantoin $(\mathrm{F})$,

Sulphamethaxazole(SXT), Erythromycin (E), penicillin (P), Cloxacillin ( OB), Neomycin (N). 
ISSN- 2350-0530(O), ISSN- 2394-3629(P)

https://doi.org/10.29121/granthaalayah.v8.i4.2020.20

In our study 99.3\%, isolates of E.coli were multidrug resistant (Table4, fig1), only one isolate was resistant to two antibiotics. This result is consistent with the result of Shafaq et al., (2011), who reported that all E.coli Strains in their study were multidrug resistant. This is quite high when compared to other studies. Prevalence of MDR E.coli was about $76.5 \%$ in a study done by Niranjan and Malini 2014; and 52.9\% in study done by Hasan et al., 2007only 8.4\% and 7.1\% MDR E.coli reported by Mathai et al., 2008 and Sahm et al., 200.In this study 95\% of E.coli isolates were resistant to more than 3 antibiotics belonging to3 or more classes of antibiotics. However in our previous study Khamees, (2001), reported that $94.6 \%$ of E.coli strains were resistant to one antibiotic and more.

Table 4: The Frequency of Resistance Pattern of Escherichia coli Strains.

\begin{tabular}{|l|c|}
\hline Resistance Pattern & Frequency Number \\
\hline AMP-AMC-C-CR-CL-CN-TE-F-SXT-E-P-OB & 3 \\
\hline AMP-AMC-CR-CL-CN- TE-F-SXT-E-P-OB & 4 \\
\hline AMP-AMC-C-CR-CL-TE-F-SXT-E-P & 11 \\
\hline AMC-C-CR-CL-CN-TE-SXT-E-P-OB & 10 \\
\hline AMP-AMC-CR-CL-CN-SXT-E-P-OB & 5 \\
\hline AMP-AMC-C-CR-CL-CN-E-P-OB & 6 \\
\hline AMP-C-CR-CL-CN-TE-SXT-E-P & 6 \\
\hline AMC-CR-C-CL-CN-SXT-F-P & 5 \\
\hline AMP-CR-CL-CN-SXT-E-P-OB & 10 \\
\hline AMP-AMC-CR-CN-SXT-P-OB & 8 \\
\hline AMP-AMC-C-CR-E-P-OB & 6 \\
\hline AMC-CR-CL-CN-TE-E-P & 5 \\
\hline AMP-CR-CL-CN-TE-SXT-P & 6 \\
\hline AMP-AMC-CL-TE-E-P-OB & 7 \\
\hline AMP-CR-CL-CN-TE-P & 9 \\
\hline AMP-CL-CN-SXT-E-P & 10 \\
\hline AMP-CL-F-SXT-E-P & 10 \\
\hline
\end{tabular}

The increasing prevalence multidrug resistant pathogens is of considerable concern and worrisome as being public health and therapeutic challenge to clinical all over the world. Most encountered gram negative pathogens e.g. Klebsiella pneumonia and E.coli have the ability to acquire cross resistance to several antimicrobial agents (Khamees and Ghafir, 2017).In our study 60\% of E.coli isolates showed multidrug resistance to 5-8 antibiotics ( belong in to 3 or $<3$ different classes of drugs). F.g-1

Table 5: The resistance patterns of Escherichia coli strains to more than one drug

\begin{tabular}{|c|c|c|}
\hline Resistance patterns (number of drugs) & Frequency & Total No (\%) \\
\cline { 1 - 2 } $\mathbf{2}$ & 1 & \multirow{2}{*}{$20(12)$} \\
\hline $\mathbf{3}$ & 7 & \\
\hline $\mathbf{4}$ & 12 & \multirow{2}{*}{$97(60)$} \\
\hline $\mathbf{5}$ & 21 & \\
\cline { 1 - 2 } $\mathbf{6}$ & 29 & \\
\hline $\mathbf{7}$ & 24 & \\
\hline $\mathbf{8}$ & 23 & \\
\hline
\end{tabular}




\begin{tabular}{|c|c|c|}
\hline $\mathbf{9}$ & 17 & \multirow{3}{*}{$45(28)$} \\
\hline $\mathbf{1 0}$ & 21 & \\
\hline $\mathbf{1 1}$ & 3 & \\
\hline $\mathbf{1 2}$ & 3 & \\
\hline
\end{tabular}

These results are comparable to those studies found in Sudan by Ahmed et al., 2000; and Rabia et al., 2012 in Pakistan. Also 28\% of the isolates from our study showed resistance to more than 8 drugs similarly (AL-Mardeni et al., 2009) in Jordan reported that 59.9\% of E.coli isolates were resistant to three or more antibiotics. In addition, a study done in Saudi Arabia by Al-Tawafiq., (2006) reported $29 \%$ E.coli isolates were resistant to more than two antibiotics. However in study done by (Saeed et al.,2009) in Karachi, Pakistan 92\% of gram negative clinical isolates including E.coli resistant to one or more antibiotics. All these results from different countries report more or less the same results.

\section{Conclusion}

A total of 162 E.coli strains were isolated from UTI positive urine samples . 99.3\% of these isolated were multidrug resistant to 3 antibiotics and more from different classes only one strain was resistant to two antibiotics different resistant pattern occurred 3-10 times obtained.

\section{References}

[1] Ahmed, A.A., Osman, H., Mansour, A.M., MUSA, H.A., Ahmed, A.B., Karrar, Z. and Hassan, H.S., 2000. Antimicrobial agent resistance in bacterial isolates from patients with diarrhea and urinary tract infection in the Sudan. Am. J. trop. Med. Hyg., 63: 259-263.

[2] Alessandra Caracciolo, Alberto Bettinelli, Claudio Bonato, ClementinaIsimbaldi, Alessandro Tagliabue, Laura Longoni, and Mario G Bianchetti. 2011: Antimicrobial resistance among Escherichia coli that cause childhood community-acquired urinary tract infections in Northern Italy. Ital J Pediatrv.37; PMC3023741.

[3] AL-Mardeni, R.I., Batarseh, A., Omaish, L., Shraideh, M., Batarseh, B. and Unis, N., 2009. Empirical treatment for pediatric urinary tract infection and resistance patterns of uropathogens, in Queen Alia Hospital and Prince A’Isha Military Center Jordan. Saudi J. Kidney Dis. Transpl. 20: 135-139.

[4] AL-Tawfiq, J.A., 2006. Increasing antibiotic resistance among isolates of Escherichia coli recovered from inpatients and outpatients in a Saudi Arabian hospital infection control and hospital epidemiology. Infect. Control Hosp. Epidemiol., 27: 748-753.

[5] Barry, A. L. The antimicrobial susceptibility test: principles and practices pp. 180-195. Lea \& Febiger, Philadelphia, U. S. A. 1976.

[6] Dulawa J. Urinary tract infection-2003, 2004. Ann Acad Med Bialostoc. 49: 182-184.

[7] Helms M, Vastrup P, Gerner-Smidt P, Mølbak K.2002. Excess mortality associated with antimicrobial drug-resistant Salmonella typhimurium. Emerg Infect Dis. 8(5):490- 5.

[8] Hasan AS, Nair D, Kaur J, Baweja G, Deb M, Aggarwal P. 2007. Resistance patterns of urinary isolates in a tertiary Indian hospital. J Ayub Med Coll Abbottabad. 19:39-41.

[9] Jigna Naik and Pratibha Desai. 2012. Antibiotic resistance pattern in urinary isolates of Escherichia coli with special reference to extended spectrum $\beta$-Lactamases production. International Journal of pharmacy \& Life Sciences [Naik\& Desai, 3(3).

[10] Karlowsky JA, Kelly LJ, Thornberry C, Jones ME, Sahm DF. 2002. Trends in Antimicrobial Resistance among Urinary Tract Infection Isolates of Escherichia coli from Female Outpatients in the United States. Antimicrobial Agents Chemotherapy. 46(8): 2540-5. 
ISSN- 2350-0530(O), ISSN- 2394-3629(P)

https://doi.org/10.29121/granthaalayah.v8.i4.2020.20

[11] Khamees, Samiya Sabea; Ghafir, Khadija Saeed Ali. 2017. The Resistance Patterns of Multidrug Resistant Klebsiella pneumonia Isolated from Different Clinical Samples. International Journal of Pharmacy \& Life Sciences. Vol. 8 Issue 2, p5439-5445. 6p.

[12] Mathai E, Chandy S, Thomas K, Antoniswamy B, Joseph I, Mathai M, et al. 2008. Antimicrobial resistance surveillance among commensal Escherichia coli in rural and urban areas in Southern India. Trop Med Int Health. 13:41-5.

[13] Mehrgan, H. and Rahbar, M. 2008. Prevalence of extended spectrum $\beta$-lactamase-producing in a tertiary care hospital in Tehran, Iran. Int. J Antimicrobial Agents. 31: 147-151.

[14] Mejbah Uddin Ahmed, MdKhairuzzaman, Afroza Begum, Iftikhar Ahmed. 2011 Isolation and Antimicrobial Susceptibility Pattern of Escherichia coli Causing Urinary Tract Infection in Enam Medical College Hospital. J Enam Med Col 2011; 1(2): 60-62.

[15] Mohammad Alshara. Antimicrobial resistant pattern of Escherichia coli strains isolated from pediatric patients in Jordan. 2010. ActaMedicaIranica 2011; 49(5): 293-295.

[16] National Committee for Clinical Laboratory Subcommittee antimicrobial susceptibility tests: performance standards for antimicrobial disc susceptibility tests. NCCLS, Villanova A. 1990, 9.

[17] Niranjan V, Malini A. 2014. Antimicrobial resistance pattern in Escherichia coli causing urinary tract infection among inpatients. Indian J Med Res.139(6):945-8.

[18] Noor, N. Ajaz, M. Rasool, SA. and Pirzada, ZA. 2004. Urinary Tract infections associated with multidrug resistant Enteric Bacilli: Characterization and Genetical Studies. Pakistan J. Pharm. Sci. $17: 115-123$.

[19] Osterblad et al. (2000). A between species comparison of antimicrobial resistance in Enterobacteria in fecal flora. J antimicrob.Chemother; 44:1479-1484.

[20] Pfaller MA, Jones RN. (2000). MYSTIC (Meropenem Yearly Susceptibility Test Information Collection) results from the Americas: resistance implications in the treatment of serious infections. J Antimicrobial Chemotherapy; 46(Suppl):25-37.

[21] Rabia Tanvir, Rubeena Hafeez and Shahida Hasnain, 2012. Prevalence of Multiple Drug Resistant Escherichia coli in Patients of Urinary Tract Infection Registering at a Diagnostic Laboratory in Lahore, Pakistan. Pakistan J. Zool., vol. 44(3), pp. 707-712.

[22] Saeed, A., Khatoon, H. and Ansari, F.A., 2009. Multidrug resistant gram-negative bacteria in clinical isolates from Karachi. Pak. J. pharmaceut. Sci., 22: 44-48.

[23] Sahm, DF. Thornberry, C., Mayfield, D C., Jones, ME. and Karlowsky, J. 2001. Multidrug resistant urinary tract isolates of Escherichia coli: Prevalence and patients demographics in the United States in 2000. Antimicrob. Agents Chemother. 35: 235-236.

[24] Samia S. Khamees. 2001. Resistance Patterns of Escherichia Coli Causing Urinary Tract Infection Antibiotics. Kufa Med. J. Vol.4. No. 1.

[25] Semra K, Suheyla S, Cenk S, Horu G, Mehmet G, Beril O, 2005. Increasing antimicrobial resistance of Escherichia coli isolates from community acquired UTI during 1998-2003 in Manisa, Turkey. Japan Journal of Infectious Disease.,58: 159-161.

[26] Shafaq Aiyaz Hassan, Syed Asfar Jamal and Mustafa Kamal. 2011. Occurrence of multidrug resistant and Esbl Producing E.coli Causing Urinary Tract Infections. Journal of Basic and Applied Sciences Vol. 7, No. 1, 39-43.

[27] Taneja, N. Rao, P. Arora, J. Ashok, DA. 2008. Occurrence of ESBL and Amp-C $\beta$-lactamases \& susceptibility to newer antimicrobial agents in complicated UTI. Ind. J. Med. Res. 127: 85-88.

[28] Tanzina Akter, Mohammad Jakir Hossain, MdSumon Khan, Hoomyra Sultana, Kaniz Fatema, Sohana Al Sanjee, Suvamoy Datta. 2016. Isolation, identification and antimicrobial susceptibility pattern analysis of Escherichia coli isolated from clinical samples of Bangladesh. Asian Journal of Biomedical and Pharmaceutical Sciences. 6 (54), 13-16.

* Corresponding author.

E-mail address: alwan_sami2005@yahoo.com 